\title{
Estimation of the orientation term of the general quadrature transform from a single $n$-dimensional fringe pattern
}

\author{
J. Antonio Quiroga \\ Departamento de Óptica, Facultad de Ciencias Físicas, Universidad Complutense, Ciudad Universitaria s/n, \\ 28040 Madrid, Spain \\ Manuel Servin \\ Centro de Investigaciones en Optica AC, P.O. Box 1-948, León 37150, Guanajuato, México \\ J. Luis Marroquin \\ Centro de Investigación en Matemáticas, P.O. Box 402, Guanajuato 37000, Guanajuato, México \\ Daniel Crespo \\ Departamento de Óptica, Facultad de Ciencias Físicas, Universidad Complutense, Ciudad Universitaria s/n, \\ 28040 Madrid, Spain
}

Received June 4, 2004; revised manuscript received August 26, 2004; accepted September 21, 2004

\begin{abstract}
The spatial orientation of the fringe has been demonstrated to be a key point in the reliable phase demodulation from a single $n$-dimensional fringe pattern regardless of the frequency spectrum of the signal. The recently introduced general $n$-dimensional quadrature transform (GQT) makes explicit the importance of the fringe orientation in the demodulation process. The GQT is a quadrature operator that transforms cos $\phi$ into $-\sin \phi$-where $\phi$ is the modulating phase-and it is composed of two terms: an orientation factor directly related to the fringe's spatial orientation and an isotropic $n$-dimensional generalization of the one-dimensional Hilbert transform. We present a method for the determination of the orientation factor in a general $n$-dimensional case and its application to the demodulation of a single fringe pattern by the GQT. We have tested the algorithm with simulated as well as real photoelastic fringe patterns with good results. (C) 2005 Optical Society of America
\end{abstract}

OCIS codes: $100.0100,100.2650,100.6890,120.5050$.

\section{INTRODUCTION}

The irradiance distribution of a fringe pattern can be represented in a first approximation as an $n$-dimensional phase-modulated signal given by

$$
I(\mathbf{r})=b(\mathbf{r})+m(\mathbf{r}) \cos \phi(\mathbf{r}),
$$

where $I$ is the irradiance, $b$ is the background, $m$ is the modulation, $\phi$ is the modulating phase, and $\mathbf{r}$ $=\left(x_{1}, \ldots, x_{n}\right)$ denotes an $n$-dimensional position vector [for example, in a temporal experiment in which a series of images are obtained, $n=3$ and $\mathbf{r}=\mathbf{r}(x, y, t)$, with $t$ as the time and $x, y$ as the spatial coordinates]. Usually, in this model the modulating phase is associated with the physical magnitude to be measured, and the background and the modulation are associated with environmental conditions, such as the illumination setup or object reflectance or both.

Phase demodulation from a single fringe pattern is an important task in areas such as the study of fast transient phenomena, where only one fringe pattern can be acquired (two-dimensional case), or a temporal set can be captured but with unknown phase temporal behavior [three-dimensional (3D) case]. In the general case of fringe patterns with closed fringes, it is not possible to obtain good results with ordinary algorithms such as the Fourier transform or spatial phase-sampling methods. ${ }^{1}$ To solve this problem, researchers have developed several methods. $^{2-5}$

Recently two quadrature methods have appeared, based on the isotropic generalization of the onedimensional Hilbert transform for the phase demodulation from a single fringe pattern, ${ }^{4,5}$ in which the importance of the fringe orientation is made explicit, appearing as a term of the quadrature operator.

The aim of any $n$-dimensional quadrature operator $Q_{n}\{\cdot\}$ is to transform a given single fringe pattern into its quadrature image. If we assume that the background is spatially smooth, we can remove it by using a high-pass filter. If we denote by $I_{\mathrm{HP}}$ the high-pass-filtered background-suppressed version of the irradiance signal given by Eq. (1), then $I_{\mathrm{HP}}(\mathbf{r})=m(\mathbf{r}) \cos \phi(\mathbf{r})$ and $Q_{n}\left\{I_{\mathrm{HP}}(\mathbf{r})\right\}=-m(\mathbf{r}) \sin \phi(\mathbf{r})$. With this quadrature sig- 
nal, one can easily determine the modulo $2 \pi$ wrapped phase, $W\{\phi(\mathbf{r})\}$, over the whole region of interest by a simple arctangent calculation.

The general quadrature transform (GQT) presented in Ref. 5 is a quadrature operator given by

$$
Q_{n}\left\{I_{\mathrm{HP}}(\mathbf{r})\right\}=\frac{\nabla \phi(\mathbf{r})}{|\nabla \phi(\mathbf{r})|} \cdot \frac{\nabla I_{\mathrm{HP}}(\mathbf{r})}{|\nabla \phi(\mathbf{r})|}=\mathbf{n}_{\phi}(\mathbf{r}) \cdot \frac{\nabla I_{\mathrm{HP}}(\mathbf{r})}{|\nabla \phi(\mathbf{r})|} .
$$

From Eq. (2) it is possible to see that the GQT is composed of two terms. The second term is an isotropic $n$-dimensional generalization of the one-dimensional Hilbert transform, $\mathbf{H}_{n}\left\{I_{\mathrm{HP}}\right\}=\nabla I_{\mathrm{HP}} /|\nabla \phi|$. The first term of Eq. (2), $\mathbf{n}_{\phi}(\mathbf{r})=\nabla \phi(\mathbf{r}) /|\nabla \phi(\mathbf{r})|$, is a unit vector normal to the corresponding isophasics, which points in the direction of the $\nabla \phi(\mathbf{r})$-denominated fringe orientation term. In $n$ dimensions it is worthwhile to express $\mathbf{n}_{\phi}$ as a function of its direction cosines:

$$
\mathbf{n}_{\phi}(\mathbf{r})=\frac{\nabla \phi(\mathbf{r})}{|\nabla \phi(\mathbf{r})|}=\sum_{k=1}^{n} c_{k}^{(2 \pi)} \mathbf{e}_{k}
$$

where $\mathbf{e}_{k}, k=1 \ldots n$, is an orthonormal base in the direct space and $c_{k}^{(2 \pi)}$ are the direction cosines given by

$$
c_{k}^{(2 \pi)}=\cos \alpha_{k}^{(2 \pi)}=\frac{\partial \phi / \partial x_{k}}{|\nabla \phi(\mathbf{r})|}=\frac{\omega_{k}}{|\nabla \phi(\mathbf{r})|},
$$

where $\alpha_{k}^{(2 \pi)}, k=1 \ldots n$, are the angles between the $k$ th coordinate axis and $\nabla \phi(\mathbf{r})$ and $\omega_{k}$ can be interpreted as the local spatial frequencies of the fringe pattern. The superscript $(2 \pi)$ is used because in two dimensions $\alpha_{k}^{(2 \pi)}$ corresponds to the modulo $2 \pi$ fringe's orientation angle. ${ }^{6}$

In consequence, to demodulate a single $n$-dimensional fringe pattern by the GQT, it is necessary to compute $\mathbf{H}_{n}\left\{I_{\mathrm{HP}}\right\}$ and the orientation term $\mathbf{n}_{\phi}(\mathbf{r})$. The estimation of $\mathbf{H}_{n}\left\{I_{\mathrm{HP}}\right\}$ as a linear operator regardless of the dimension of the problem is shown in Ref. 5. However, the computation of the orientation term has been demonstrated only up to two dimensions. ${ }^{6}$ In effect, in two dimensions there is a clear correlation between $\alpha_{1}^{(2 \pi)}$ and $\alpha_{2}^{(2 \pi)}$, given by $\alpha_{1}^{(2 \pi)}-\alpha_{2}^{(2 \pi)}=\pi / 2$, that easily allows their correct computation from the irradiance gradient. However, for dimensions higher than two, this correlation is not so obvious; this is a key point of the GQT method because without the possibility of the orientation term computation the generalization represented by the GQT is not possible. In Section 2 we will show how the orientation term can be computed in a general $n$-dimensional case from a single fringe pattern given by Eq. (1), making possible the application of the GQT demodulation method to an $n$-dimensional case.

\section{2. n-DIMENSIONAL ORIENTATION TERM COMPUTATION}

In the case of fringe patterns, as the phase is wrapped by the observation process, it is not possible to find a linear system to calculate the field $\mathbf{n}_{\phi}$ for an image with closed fringes. To obtain the orientation term, we have access only to the fringe pattern's irradiance and its gradient, from which it is possible to compute a new set of direction cosines given by

$$
c_{k}=\frac{\partial I / \partial x_{k}}{|\nabla I(\mathbf{r})|}
$$

If the modulation $m(\mathbf{r})$ and the background $b(\mathbf{r})$ are spatially smooth, we can approximate $\nabla I \approx-m \sin \phi \nabla \phi$, and Eq. (5) becomes

$$
\begin{aligned}
c_{k} & =\frac{-\sin \phi}{|\sin \phi|} \frac{\partial \phi / \partial x_{k}}{|\nabla \phi|} \\
& =-\operatorname{sgn}(\sin \phi) \frac{\partial \phi / \partial x_{k}}{|\nabla \phi|}=-\operatorname{sgn}(\sin \phi) c_{k}^{(2 \pi)} .
\end{aligned}
$$

Therefore if we compute the orientation term from the irradiance gradient instead of the phase gradient, we obtain

$$
\tilde{\mathbf{n}}_{\phi}(\mathbf{r})=\frac{\nabla I}{|\nabla I|}=\sum_{k=1}^{n} c_{k} \mathbf{e}_{k}=-\operatorname{sgn}(\sin \phi) \mathbf{n}_{\phi}(\mathbf{r}) .
$$

If the modulation and background were not spatially smooth, the approximation $\nabla I \approx-m \sin \phi \nabla \phi$ will not hold, and Eq. (6) will not describe the problem correctly. In this case, the main consequence is that the orientation term given by Eq. (7) will not have the correct magnitude and will not point in the correct direction. ${ }^{7}$ This is usually the case in experimental techniques as holographic interferometry, photoelasticity, and shadow moiré. In this case the use of a normalization technique (background suppression and modulation equalization) is recommended. For the examples presented in this paper we have used an isotropic $n$-dimensional normalization. ${ }^{8}$ This method is based on the use of the so-called quasiquadrature operator. As is demonstrated in Ref. 8, from

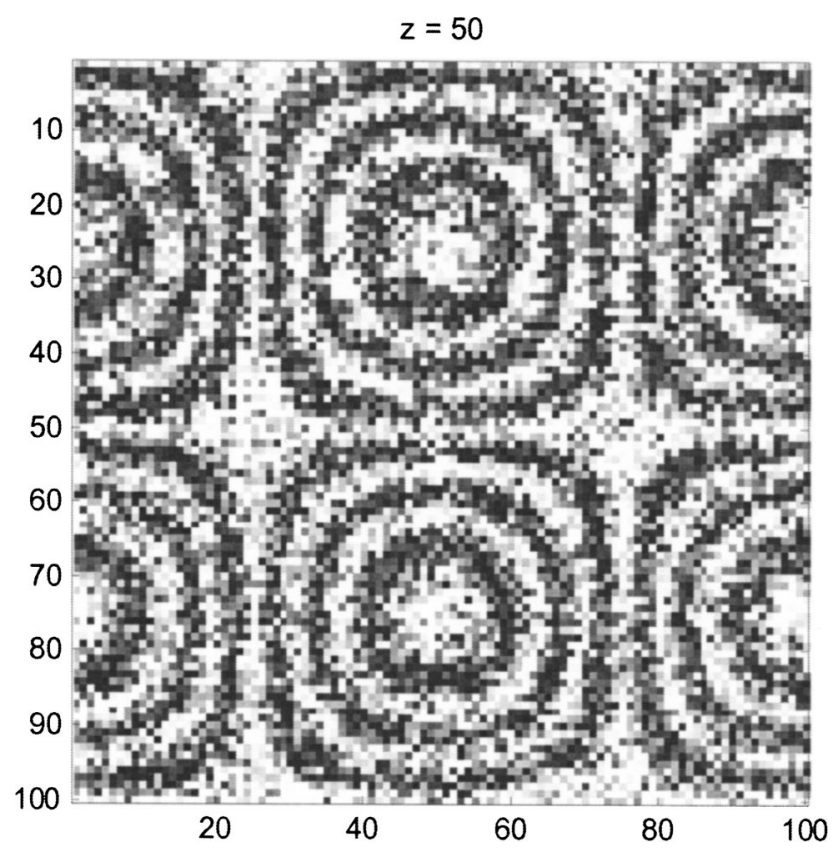

Fig. 1. Noisy fringe pattern with additive phase noise with an image size of $100 \times 100$ pixels. 


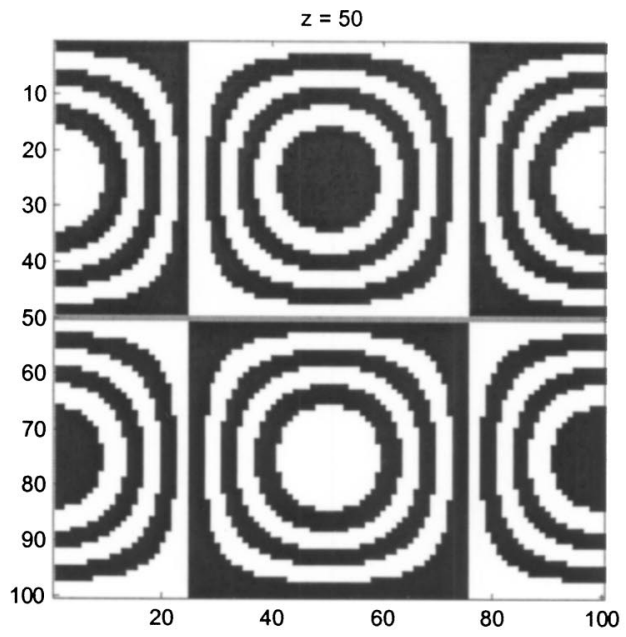

(a)

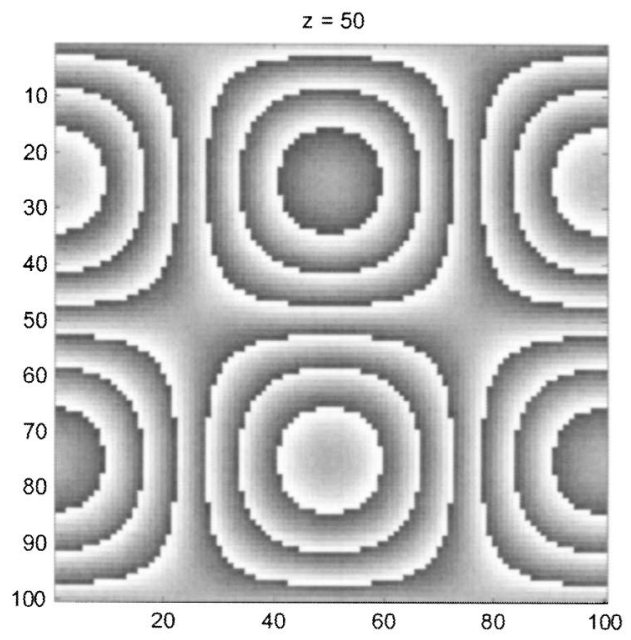

(c)

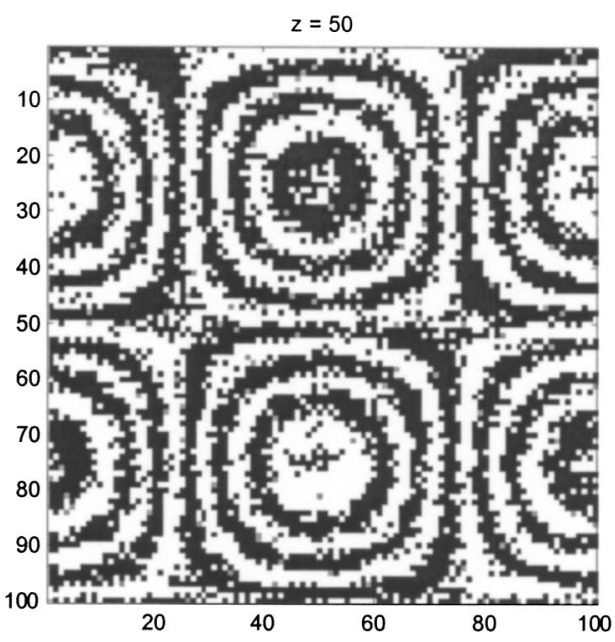

(b)

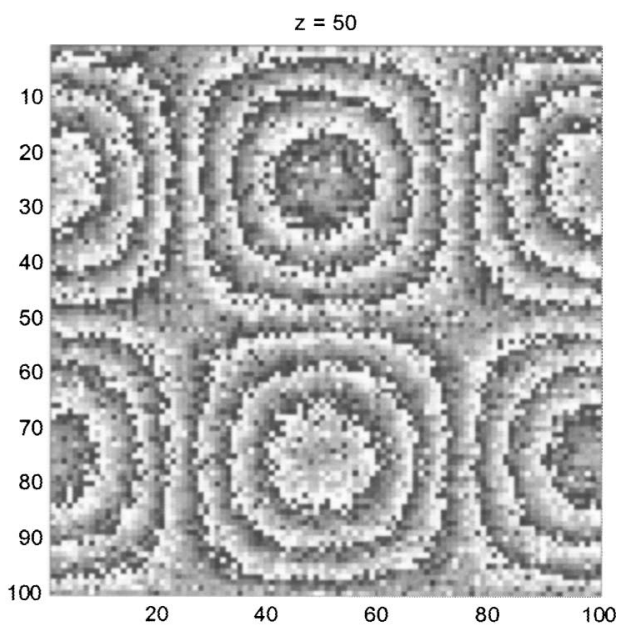

(d)

Fig. 2. (a) Theoretical QS for the fringe pattern depicted in Fig. 1, (b) corresponding QS obtained by the proposed method, (c) theoretical modulating wrapped phase of the fringe pattern shown in Fig. 1, (d) wrapped phase obtained by the GQT with the QS depicted in Fig. 2(b). For the QS images, black corresponds to -1 and white to 1 . For the wrapped phase images, black corresponds to 0 and white to $2 \pi$.

the quasi-quadrature operator given by $\widetilde{Q}_{n}\left\{I_{\mathrm{HP}}(\mathbf{r})\right\}$ $=\widetilde{\mathbf{n}}_{\phi}(\mathbf{r}) \cdot \nabla I_{\mathrm{HP}}(\mathbf{r}) /|\nabla \phi(\mathbf{r})|$, it is possible to compute a phase map, $W\{\tilde{\phi}\}$, from which the normalized fringe pattern is computed as $I_{N}(\mathbf{r})=\cos [W\{\tilde{\phi}(\mathbf{r})\}]=\cos \phi(\mathbf{r})$.

Equation (7) states that we can compute the orientation term $\mathbf{n}_{\phi}$ from the irradiance gradient if we can estimate the fringe-pattern quadrature sign (QS) given by

$$
\mathrm{QS}\{I\}=-\operatorname{sgn}(\sin \phi) .
$$

This is the main idea of the proposed method: If we can estimate the QS of a fringe pattern, we will be able to compute the correct orientation term independently of the dimension of the problem.

To compute the QS in a general case, we propose the following method. First we will define a set of modulo $2 \pi$ angles given by

$$
\beta_{k}^{(2 \pi)}=\arctan \left(\frac{-\partial \phi / \partial x_{k+1}}{\partial \phi / \partial x_{k}}\right)=\arctan \left(\frac{-\omega_{k+1}}{\omega_{k}}\right),
$$

where $k=1 \ldots n$ and $k+1$ is a circular operation, that is, $n+1 \equiv 1$. Geometrically, $\beta_{k}^{(2 \pi)}$ are the modulo $2 \pi$ angles subtended by the phase-gradient projection on the $(k, k+1)$ plane with the $k$ th coordinate axis. As before, we have no access to the phase gradient but only to the irradiance gradient; then what we can calculate directly are the angles given by

$$
\beta_{k}^{(\pi)}=\arctan \left(\frac{-\partial I / \partial x_{k+1}}{\partial I / \partial x_{k}}\right),
$$

which, owing to the sign flips of the irradiance gradient, are defined modulo $\pi .^{6,7}$ The cosines of the angles defined by Eqs. (9) and (10) are

$$
\begin{aligned}
\cos \beta_{k}^{(2 \pi)} & =\frac{\omega_{k}}{\left(\omega_{k}^{2}+\omega_{k+1}^{2}\right)^{1 / 2}}, \\
\cos \beta_{k}^{(\pi)} & =\frac{\partial I / \partial x_{k}}{\left[\left(\partial I / \partial x_{k}\right)^{2}+\left(\partial I / \partial x_{k+1}\right)^{2}\right]^{1 / 2}} .
\end{aligned}
$$


If the modulation and the background are smooth functions, the irradiance gradient can be approximated by $\nabla I \approx-m \sin \phi \nabla \phi$, and therefore these cosines are related by

$$
\cos \beta_{k}^{(\pi)}=-\operatorname{sign}[\sin \phi] \cos \beta_{k}^{(2 \pi)}=\mathrm{QS}\{I\} \cos \beta_{k}^{(2 \pi)} .
$$

Finally, Eq. (13) indicates that if we are able to calculate $\cos \beta_{k}^{(2 \pi)}$ we can compute the QS in the general $n$-dimensional case from the direct measurement $\cos \beta_{k}^{(\pi)}$ as

$$
\begin{aligned}
\mathrm{QS}\{I\} & =\operatorname{sgn}\left(\cos \beta_{k}^{(\pi)}\right) \operatorname{sgn}\left(\cos \beta_{k}^{(2 \pi)}\right) \\
& =\operatorname{sgn}\left(\partial I / \partial x_{k}\right) \operatorname{sgn}\left(\cos \beta_{k}^{(2 \pi)}\right),
\end{aligned}
$$

where we have used the properties of the signum function and the fact that, from Eq. (12), $\operatorname{sign}\left(\cos \beta_{k}^{(\pi)}\right)$ $=\operatorname{sign}\left(\partial I / \partial x_{k}\right)$.

The $\beta_{k}^{(\pi)}$ fields are, by definition, noisy and piecewise discontinuous in the case of real fringe patterns with closed fringes; however, it is possible to reliably compute

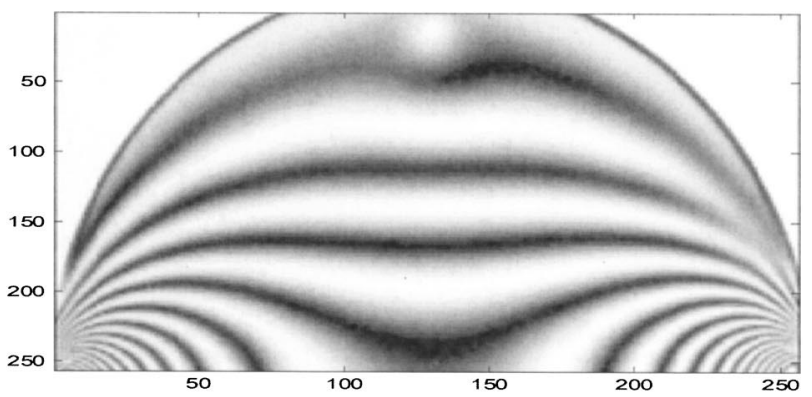

(a)

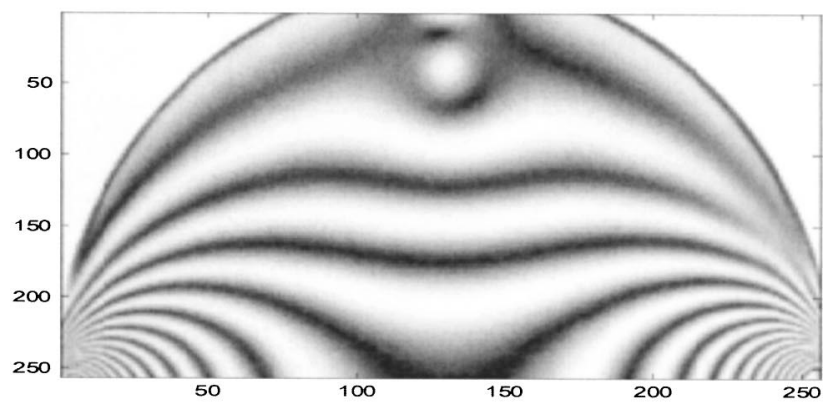

(b)

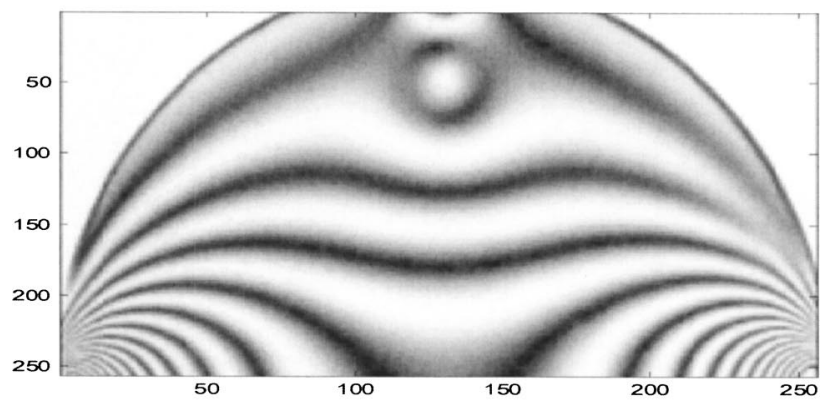

(c)

Fig. 3. (a)-(c) Circular bright-field isochromatic fringe patterns of a stress-frozen diametrically compressed disk. The increasing load between snapshots is applied in the upper part of the figures.

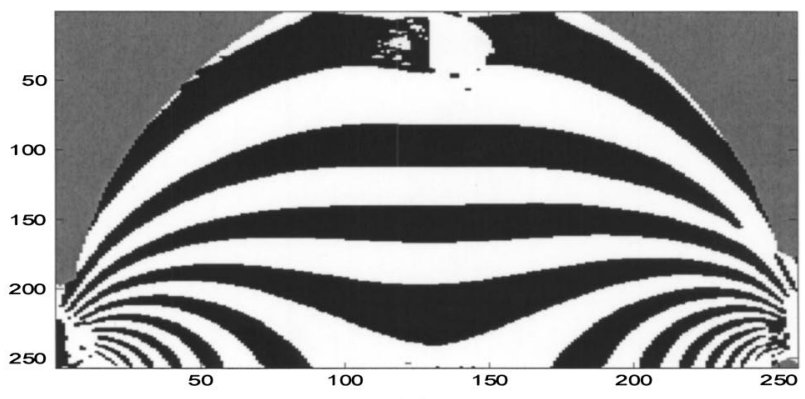

(a)

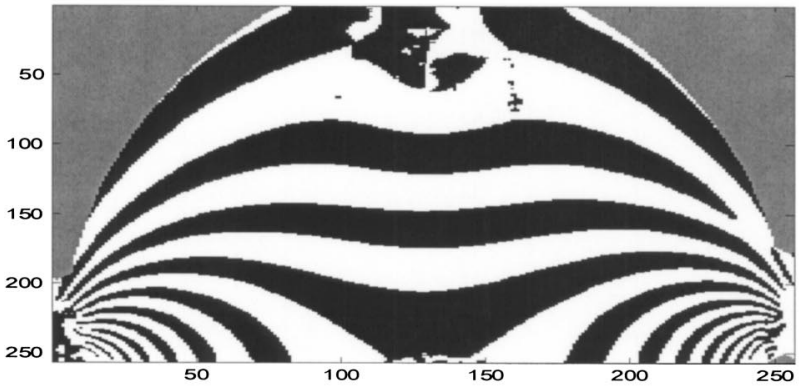

(b)

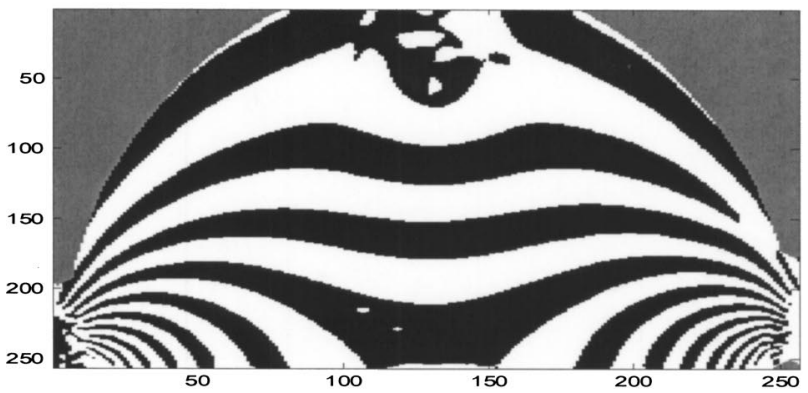

(c)

Fig. 4. (QS) obtained by the proposed method for each of the three images depicted in Fig. 3. In this figure, black corresponds to -1 , white to 1 , and gray to nonprocessed.

$\beta_{k}^{(2 \pi)}$ from direct measurements of $\beta_{k}^{(\pi)}$ by use of the phase-unwrapping technique described in Ref. 6. For the sake of clarity, we will include a brief discussion of the method. The relation of $\beta_{k}^{(\pi)}$ to $\beta_{k}^{(2 \pi)}$ is $\beta_{k}^{(\pi)}=\beta_{k}^{(2 \pi)}$ $+l \pi$, where $l$ is an integer such that $0 \leqslant \beta_{k}^{(\pi)} \leqslant \pi$, therefore $\quad W\left\{2 \beta_{k}^{(\pi)}\right\}=W\left\{2 \beta_{k}^{(2 \pi)}+2 l \pi\right\}=W\left\{2 \beta_{k}^{(2 \pi)}\right\}$. Thus by unwrapping the phase map $W\left\{2 \beta_{k}^{(\pi)}\right\}$, one can obtain $\beta_{k}^{(2 \pi)}$ and therefore the QS.

In the two-dimensional case, by definition, $\alpha_{k}^{(\pi)}=\beta_{k}^{(\pi)}$ and $\alpha_{k}^{(2 \pi)}=\beta_{k}^{(2 \pi)}$; thus the computation of the modulo $2 \pi$ fringe's orientation angle, $\alpha_{1}^{(2 \pi)}$, and the QS are equivalent. For dimensions greater than two, it makes no sense to speak about the modulo $2 \pi$ fringe's orientation angle; however, the QS and the direction cosines (and therefore the orientation term) are always well defined.

Once the QS is computed by Eq. (14), the orientation term $\mathbf{n}_{\phi}$ can be calculated from the irradiance by Eq. (7), the GQT can be applied to obtain the quadrature term according to Eq. (2), and therefore the fringe-pattern modulating phase can be obtained.

In finishing this section, it is worth mentioning that the QS is by definition—see Eq. (8)—unique and independent 
of the index $k$. Therefore it is not necessary to compute the QS for each component $k$ of the fringe pattern; with Eq. (14), only one phase-unwrapping process of $2 \beta_{k}^{(\pi)}$ for a given $k$ is necessary. However, noise can produce local

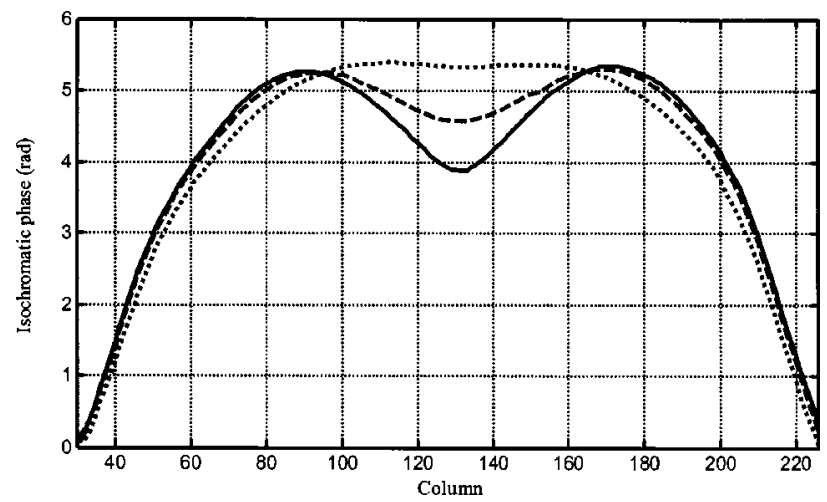

Fig. 5. Profiles along line 90 of the demodulated phase corresponding to the three images depicted in Fig. 3. Dotted, dashed, and solid curves correspond to initial, intermediate, and final states, respectively.

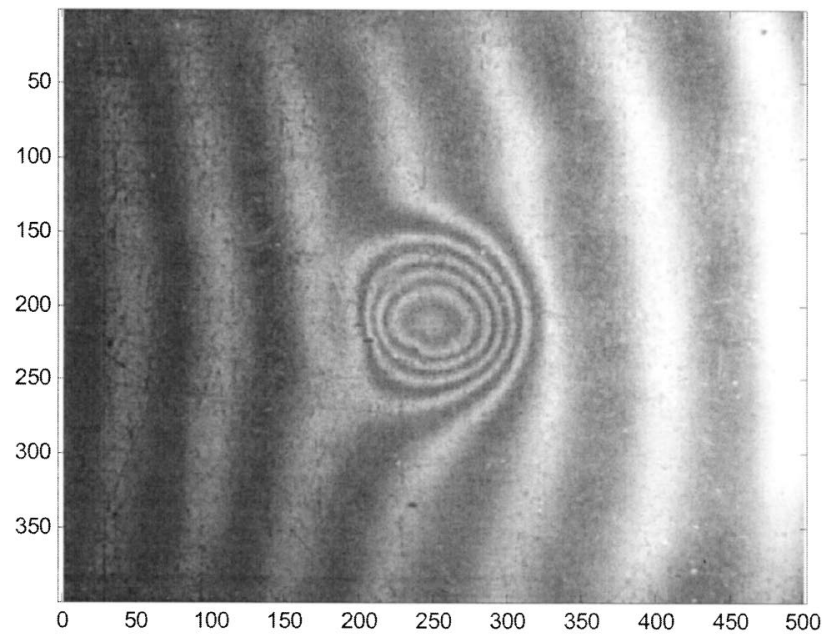

(a)

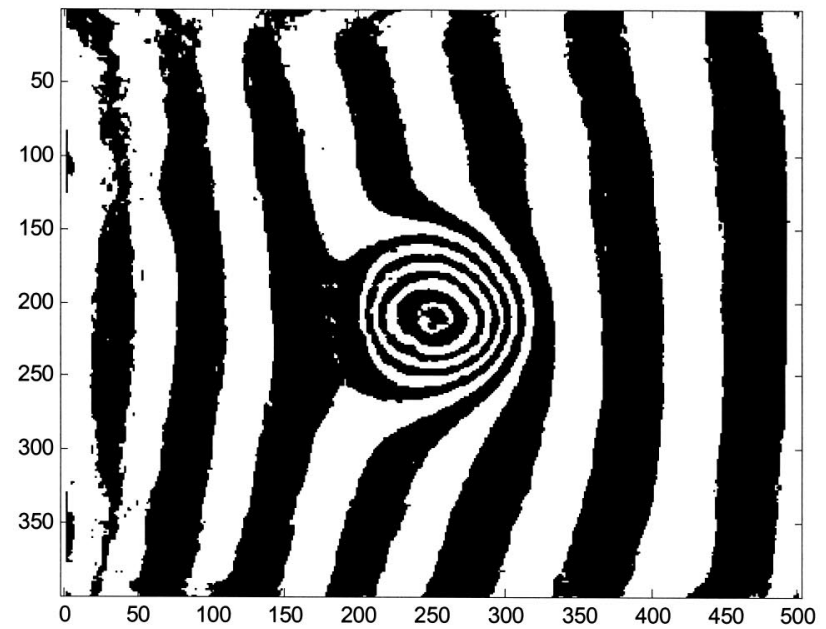

(c)

Fig. 6. (a) Shadow-moiré fringe pattern of a 350- $\mu \mathrm{m}$ indentation. QS obtained by the proposed method. (d) Recovered phase map. failures in the unwrapping process that will make the recovered QS incorrect in some places. In this case it is always possible to obtain as many as $n$ independent measurements of the QS (one for every index value) that can help to make the demodulation process more reliable in the presence of noise.

\section{EXPERIMENTAL RESULTS}

To test the procedure described above, we used a 3D computer-generated fringe pattern given by $I(\mathbf{r})$ $=\operatorname{round}\{128+100 \cos [\phi(\mathbf{r})+f(\mathbf{r})]\}$ with $\phi(\mathbf{r})$ $=6 \pi \cos (2 \pi x / N) \sin (2 \pi y / N) \cos (2 \pi z / N)$, where $N=100$ and $x, y, z=-N / 2 \ldots N / 2-1 ; f$ is a zero-mean, Gaussiandistributed signal with a standard deviation of $1 \mathrm{rad}$, modeling additive phase noise; $\phi$ is the modulating phase; and round $\{\cdot\}$ indicates the rounding to the nearest integer operation. Figure 1 shows the irradiance at plane $z$ $=50$, Figs. 2(a) and 2(b) show the theoretical QS calculated by Eq. (8) and the estimated QS computed by Eq. (14) for the same plane, and Figs. 2(c) and 2(d) show, respectively, the noise-free theoretical modulating phase

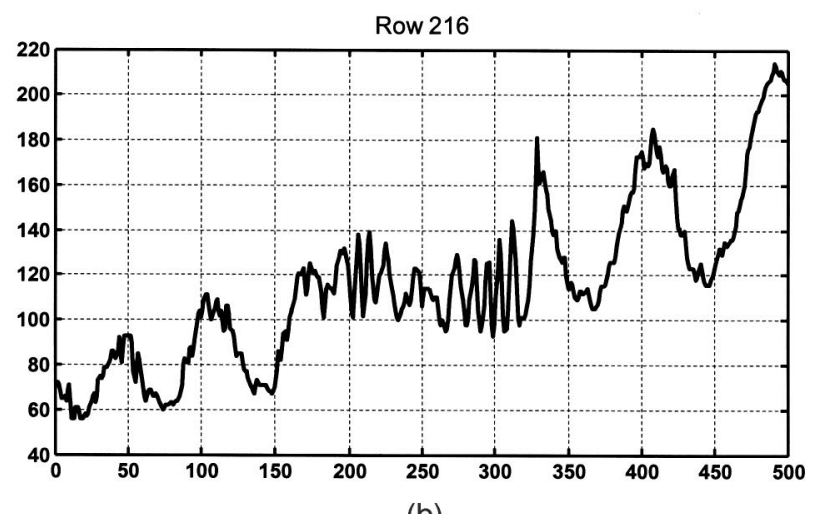

(b)

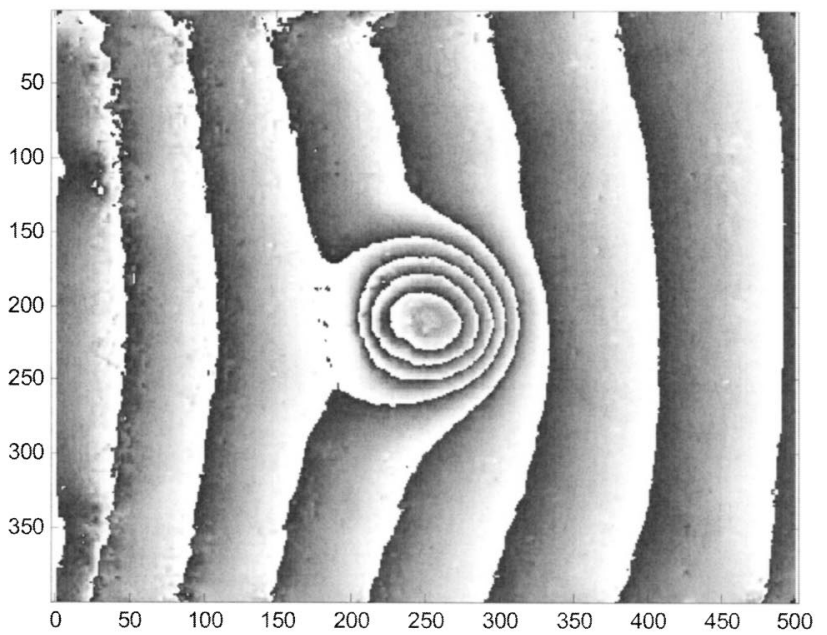

(d)

(b) Profile along row 216 of the fringe pattern shown in Fig. 6(a). (c) 
given by equation and the modulating phase obtained at plane $z=50$ by means of the GQT method.

To show the behavior of the proposed method with real images, we have demodulated the phase for an experimental temporal sequence of five photoelastic fringe patterns with dimensions of $256 \times 256$ pixels; thus the images can be considered a spatiotemporal signal with dimensions of $256 \times 256 \times 3$ pixels. The fringe patterns were obtained with a stressed transparent object placed in a circular polariscope and observed in transmission in the circular bright-field configuration; thus only halforder isochromatics are obtained ${ }^{9}$ - that is, dark fringes correspond to a retardation $\delta=(2 m+1) \pi$, with $m$ $=0,1,2 \ldots$ In particular, the specimen is a diametrically compressed stress-frozen disk to which we have applied a variable load in a point on the free circular border perpendicularly to the loading diameter. Figures 3(a)3 (c) show three of the five images with the initial, intermediate, and final states, respectively. In this example it can be seen how the fringe corresponding to $\delta=\pi$ moves down as the load increases. Figures 4(a)-4(c) show the images of the QS corresponding to Figs. 3(a)-3(c); these images have been calculated with only the $\beta_{1}^{(\pi)}$ $=\arctan (-\partial I / \partial y / \partial I / \partial x)$ angle, but in this case the other two angles given by Eq. (10) could be used to increase the reliability of the calculation. Figure 5 shows the profile of the evolution of the demodulated phase along line 90 for the three images shown in Fig. 3 (dotted, dashed, and continuous curves correspond to initial, intermediate, and final states, respectively). Two inflection points can be observed near columns 95 and 165 where the temporal rate of the phase changes its sign; between these two columns the phase decreases with the applied load, and outside them the phase increases. As can be observed, the main advantage of computing the orientation term from the 3D signal instead of processing each temporal slice is the automatic guarantee of the sign consistency for the whole 3D signal. Additionally, in this case a temporal asynchronous algorithm will be not able to process the zones corresponding to the inflection points (temporal rate near zero) and to obtain the correct rate sign at each side of the inflection points.

Finally, to show the performance of the proposed technique with an experimental fringe pattern with significant background and modulation spatial variations, we have processed a shadow-moiré fringe pattern corresponding to a indentation of $350 \mu \mathrm{m}$ with a lateral size of approximately $1 \mathrm{~cm}$. Figure 6(a) shows the experimental fringe pattern with a size of $399 \times 500$ pixels. Figure 6 (b) shows the profile corresponding to the row number 216 where variation in modulation and background can be observed (in this case the normalization method of Ref. 6 was used). Figure 6(c) shows the QS computed by the proposed technique, and, finally, Fig. 6(d) shows the de- modulated phase (modulo $2 \pi$ wrapped) corresponding to the fringe pattern depicted in Fig. 6(a).

\section{CONCLUSIONS}

We have shown that the demodulation process of a single $n$-dimensional fringe pattern by means of the general quadrature transform is basically a linear operation except for the computation of the orientation term. The computation of the orientation term reduces to the calculation of the quadrature sign, and we have shown how it can be estimated from a single $n$-dimensional fringe pattern by a phase-unwrapping process of phase maps derived from the irradiance gradient. We have applied the proposed method to simulated as well as real fringe patterns with good results.

\section{ACKNOWLEDGMENTS}

We acknowledge the economic support of this work given by project DPI2002-02104 of the Ministerio de Ciencia y Tecnología of Spain and by Consejo Nacional de Ciencia y Tecnología, Mexico. Figure 6(a) is courtesy of NDT Expert, Toulouse, France; www.ndt-expert.fr.

\section{J. Antonio Quiroga's e-mail address is aq@fis.ucm.es.}

\section{REFERENCES}

1. T. Kreis, Holographic Interferometry (Akademie Verlag, Berlin, 1996).

2. J. L. Marroquin, R. Rodriguez-Vera, and M. Servin, "Local phase from local orientation by solution of a sequence of linear systems,” J. Opt. Soc. Am. A 15, 1536-44 (1998).

3. M. Servin, J. L. Marroquin, and F. J. Cuevas, "Fringefollower regularized phase tracker for demodulation of closed-fringe interferograms," J. Opt. Soc. Am. A 18, 689695 (2001).

4. K. G. Larkin, D. J. Bone, and M. A. Oldfield, "Natural demodulation of two-dimensional fringe patterns. I. General background of the spiral phase quadrature transform," J. Opt. Soc. Am. A 18, 1862-1870 (2001).

5. M. Servin, J. A. Quiroga, and J. L. Marroquin, "General $n$-dimensional quadrature transform and its application to interferogram demodulation,” J. Opt. Soc. Am. A 20, 925934 (2003).

6. J. A. Quiroga, M. Servín, and F. J. Cuevas, "Modulo $2 \pi$ fringe orientation angle estimation by phase unwrapping with a regularized phase tracking algorithm," J. Opt. Soc. Am. A 19, 1524-1531 (2002).

7. X. Zhou, J. P. Baird, and J. F. Arnold, "Fringe-orientation estimation by use of a Gaussian gradient filter and neighboring-direction averaging," Appl. Opt. 38, 795-804 (1999).

8. J. A. Quiroga and M. Servin, "Isotropic $n$-dimensional fringe pattern normalization," Opt. Commun. 224, 221-227 (2003).

9. K. Ramesh, Digital Photoelasticity (Springer-Verlag, Berlin, 2000). 